Preprint typeset in JHEP style. - HYPER VERSION

DAMTP-2000-23

Cavendish-HEP-00/02

CERN-TH/2000-144

\title{
Naturalness Reach of the Large Hadron Collider in Minimal Supergravity
}

\author{
B.C. Allanach*, J.P.J. Hetherington ${ }^{\dagger}$, M.A. Parker ${ }^{\dagger}$ and B.R. Webber ${ }^{\dagger \dagger}$ \\ *DAMTP, University of Cambridge, Wilberforce Rd, Cambridge CB3 0WA, UK \\ ${ }^{\dagger}$ Cavendish Laboratory, University of Cambridge, Madingley Road, Cambridge, \\ CB3 OHE, UK \\ ‡Theory Division, CERN, 1211 Geneva 23, Switzerland
}

\begin{abstract}
We re-analyse the prospects of discovering supersymmetry at the LHC, in order to re-express coverage in terms of a fine-tuning parameter and to extend the analysis to scalar masses $\left(m_{0}\right)$ above $2 \mathrm{TeV}$. We use minimal supergravity (mSUGRA) unification assumptions for the SUSY breaking parameters. The discovery reach at high $m_{0}$ is of renewed interest because this region has recently been found to have a focus point, leading to relatively low fine-tuning, and because it remains uncertain how much of the region can be ruled out due to lack of radiative electroweak symmetry breaking. The best fine tuning reach is found in a mono-leptonic channel, where for $\mu>0, A_{0}=0$ and $\tan \beta=10$ (within the focus point region), and a top mass of $174 \mathrm{GeV}$, all points in mSUGRA with $m_{0}<4000 \mathrm{GeV}$, with a fine tuning measure up to 210 (500) are covered by the search, where the definition of fine-tuning excludes (includes) the contribution from the top Yukawa coupling. Even for arbitrarily high $m_{0}, \mathrm{mSUGRA}$ can be discovered through gaugino events, provided the gaugino mass parameter $M_{1 / 2}<460 \mathrm{GeV}$. In this region, the mono-leptonic channel still provides the best reach.
\end{abstract}

KEYWORDs: Supersymmetry Breaking, Beyond Standard Model, Supersymmetric Standard Model, Hadronic Colliders. 


\section{Contents}

1. Introduction 1

2. Fine tuning 3

3. Radiative electroweak symmetry breaking

4. Monte-Carlo simulation of LHC discovery reach 6

5. Results 8

6. Summary 12

\section{Introduction}

A possibility for new physics beyond the standard model is supersymmetry (SUSY). If fermionic generators are added to the bosonic generators of the Lorentz group, the new space-time symmetry is supersymmetry. As a result of exact supersymmetry, all particles have a partner of equal mass but opposite spin-statistics. Cancellations between bosonic and fermionic loops prevent radiative corrections from driving scalar masses up to the highest scale present, assumed to be the GUT or Planck scale, $10^{16}$ to $10^{19} \mathrm{GeV}$, solving the naturalness problem of the standard model. In addition, the renormalised electromagnetic, weak, and strong couplings can be made to converge to an approximately common value at the grand unification scale.

Since supersymmetry is not observed amongst the already discovered particles, it must be a broken symmetry. The scale at which supersymmetry is broken, $M_{\mathrm{SUSY}}$, would be the typical mass of the as yet undiscovered superpartners of the standard model particles, and represents the scale at which this new physics becomes relevant. Considerations of general new physics beyond the standard model [四] can result in upper bounds on new physics scales of order a few TeV. However, in supersymmetric models [2], $M_{\mathrm{SUSY}}$ is expected to be at most around $1 \mathrm{TeV}$. If the SUSY breaking scale is too large, then, unless there are large cancellations between SUSY breaking parameters, electroweak symmetry breaking also will be of order $M_{\mathrm{SUSY}}$, and the $W$ and $Z$ bosons would have masses inconsistent with their measured values.

If $M_{\mathrm{SUSY}}$ is at or below the $\mathrm{TeV}$ scale, then supersymmetric particles will almost certainly be discovered at the Large Hadron Collider (LHC), being built at CERN. 
Indeed, detailed studies of how the SUSY parameters would be measured by the LHC general-purpose experiments ATLAS [3] and CMS [4] have been made [5].

The simplest possible SUSY extension of the standard model, with a superpartner for each standard model particle, and the addition of a second Higgs scalar doublet, is called the minimal supersymmetric standard model, or MSSM. The most studied sub-category of the MSSM is minimal supergravity, mSUGRA. Supergravity, where supersymmetry is a local, rather than a global symmetry, at one time motivated unification assumptions amongst the MSSM SUSY breaking parameters, reducing the number of parameters, from the hundred or so of the MSSM, to just four, plus one sign. Currently, the suppression of flavour changing neutral currents, not supergravity, is the main motivator for these assumptions. The theory is fully specified by these parameters together with those of the standard model.

As mentioned above, it is possible to avoid the problem of large electroweak boson masses if there are extra cancellations amongst the SUSY masses. However, such situations, where the parameters of a theory are carefully tuned to avoid unphysical results, are often thought to be unsatisfactory. Fundamental parameters, it is argued, should be independent, uncorrelated inputs.

These 'naturalness' arguments are often quantified in terms of 'fine tuning' [6]. There are a few different fine-tuning measures [7], and all are intended to be measures of the degree of cancellation required between fundamental parameters. A value of fine-tuning above which a theory becomes unacceptable is often advanced, and used to support the argument that $M_{\mathrm{SUSY}}$ should be small.

The authors of [8] discuss a more sophisticated measure of fine-tuning and use it to assess the status of the MSSM if superparticles are not found at the LHC. In [9] fine-tuning motivated upper bounds on MSSM masses are obtained using a complete one-loop effective potential. [10] discusses the use of fine-tuning to compare different high-energy supergravity scenarios, while [11] uses fine-tuning to compare non-minimal supersymmetric models.

Since its status as a possible solution of the naturalness problem of the standard model is one of the main reasons for investigating supersymmetry, naturalness arguments have increased relevance to studies of supersymmetry.

We therefore contend that fine-tuning is a relevant way to compare SUSY models, experiments, and search channels, and is a useful measure of experimental discovery reach. As experiments push the lower bounds on SUSY parameters upwards, the minimum fine-tuning which SUSY can have increases, and our confidence that the universe is supersymmetric at low energies falls. However, we do not believe that a high fine-tuning in itself can be used to rule a theory out.

Within mSUGRA, the fundamental SUSY breaking parameters are boundary conditions on the running SUSY breaking masses and couplings imposed at a high scale, usually taken to be $10^{16} \mathrm{GeV}$. Physical masses of superparticles are obtained by evolving the MSSM parameters to the weak scale using the renormalisation group 
equations (RGEs). The RGE evolution of minimal supergravity shows a 'focus point' behaviour [12, 13]. A relatively large region of GUT scale parameters exists for which the RGE trajectories converge towards a small range of measurable properties. Specifically, the renormalisation group trajectories of the mass squared of a Higgs doublet $\left(m_{H_{2}}^{2}\right)$ cross close to the electroweak scale.

As a result, the electroweak symmetry breaking is insensitive to the GUT scale SUSY breaking parameters [12], and fine-tuning is smaller than expected. This focus point corresponds to a region in which the scalar SUSY breaking masses, governed by the mSUGRA parameter $m_{0}$, may be large.

Previous predictions of the discovery reach of the LHC in mSUGRA parameter space, using ISAJET [14], went only as far as $m_{0}<2 \mathrm{TeV}$ [3]. The purpose of the present investigation is to extend this reach to higher $m_{0}$ and to present it in terms of a naturalness measure. We seek to determine how the standard SUSY search channels perform in this region, where charginos and neutralinos would be the dominant SUSY particles. For large $m_{0}$, squarks, sleptons, and the heavy Higgs particle could avoid detection at the LHC, and the determination of the $m_{0}$ reach of searches for these particles would be an interesting further study.

In section 2, we discuss our fine-tuning measure, and which parameters to include in its definition. We discuss in section 3 the matter of the electroweak symmetry breaking excluded region. In section 1 we discuss SUSY search channels considered for use at the LHC, and our simulation of the discovery reach using the HERWIG [15, [16] event generator. We present in section 5 the fine-tuning reach in each channel, and the overall fine-tuning reach of the LHC.

\section{Fine tuning}

At tree-level, in the MSSM, the $Z$ boson mass is determined to be [2]:

$$
\frac{1}{2} M_{Z}^{2}=\frac{m_{H_{1}}^{2}-m_{H_{2}}^{2} \tan ^{2} \beta}{\tan ^{2} \beta-1}-\mu^{2}
$$

by minimising the Higgs potential. $\tan \beta$ is the ratio of Higgs vacuum expectation values (VEVs) $v_{1} / v_{2}$ and $\mu$ is the Higgs mass parameter in the MSSM superpotential. In mSUGRA, $m_{H_{2}}$ has the same origin as the super-partner masses $\left(m_{0}\right)$. Thus as search limits put lower bounds upon super-partners' masses, the lower bound upon $m_{0}$ rises, and consequently so does $\left|m_{H_{2}}\right|$. A cancellation is then required between the terms of equation 2.1 in order to provide the measured value of $M_{Z} \ll\left|m_{H_{2}}\right|$. Various measures have been proposed in order to quantify this cancellation [7].

The definition of naturalness $c_{a}$ of a 'fundamental' parameter $a$ employed in reference [12] is

$$
c_{a} \equiv\left|\frac{\partial \ln M_{Z}^{2}}{\partial \ln a}\right| .
$$


From a choice of a set of fundamental parameters $\left\{a_{i}\right\}$, the fine-tuning of a particular model is defined to be $c=\max \left(c_{a}\right)$. Our initial choice of free, continuously valued, independent and fundamental mSUGRA parameters also follows ref. [12]:

$$
\left\{a_{i}\right\}=\left\{m_{0}, M_{1 / 2}, \mu\left(M_{\mathrm{GUT}}\right), A_{0}, B\left(M_{\mathrm{GUT}}\right)\right\}
$$

where $M_{\mathrm{GUT}} \sim 10^{16} \mathrm{GeV}$ is the GUT scale. It is this selection which gives rise to low fine-tuning for large $m_{0}$.

We have calculated $c$ numerically to one-loop accuracy in soft masses, with twoloop accuracy in supersymmetric parameters. Dominant one-loop top/stop corrections were added to the Higgs potential and used to correct eq. (2.1). The Higgs potential was minimised at $Q=\sqrt{\left(m_{\tilde{t}_{1}} m_{\tilde{t}_{2}}\right)}$, where its scale dependence is small.

Full one-loop sparticle and QCD corrections were used to determine $m_{t}\left(m_{t}\right)^{\overline{\mathrm{DR}}}[19$ and the running of $\tan \beta$ was taken into account [18] in order to calculate the Yukawa couplings from fermion running masses. Fermion running masses were determined at $M_{Z}$ by evolving them with 3-loop QCD $\otimes$-loop QED. $m_{b}\left(M_{Z}\right)^{\overline{\mathrm{DR}}}$ and $m_{\tau}\left(M_{Z}\right)^{\overline{\mathrm{DR}}}$ were determined by including one-loop SUSY QCD and third family corrections [19]. The $\overline{\mathrm{DR}}$ Higgs vacuum expectation value $v \equiv \sqrt{v_{1}^{2}+v_{2}^{2}}$ was determined by the approximate formula 19

$$
v(Q)=248.6+0.9 \ln \left(\frac{m_{\tilde{u}_{L}}}{Q}\right) \mathrm{GeV} .
$$

One-loop top, gluino and squark corrections were used [19] in order to deduce $\alpha_{S}^{\overline{\mathrm{DR}}}\left(M_{Z}\right)$ from $\alpha_{S}^{\overline{\mathrm{MS}}}\left(M_{Z}\right)=0.119$. We will examine the implications of relaxing the accuracy of the calculation below.

Note that our code does not yet include 2-loop soft terms in the RGEs, finite corrections to the electroweak gauge couplings, a full one-loop calculation of the Higgs vacuum expectation value, or tadpole contributions to the Higgs potential. It is well known [9] that improving the accuracy of the calculation of $c$ at this level can make a significant difference to its numerical value. Since its exact quantitative interpretation is obscure anyway, this fact is not in conflict with the proposed comparison of naturalness reaches in different channels.

We also consider the case where the top Yukawa coupling $h_{t}\left(M_{\mathrm{GUT}}\right)$ is added to the list of fundamental parameters in eq.(2.3). In refs. [12, 13], 20], it is shown that the focus point scenario with heavy scalars has a large fine-tuning if $h_{t}$ is included. The inclusion of $h_{t}\left(M_{\mathrm{GUT}}\right)$ into the definition of fine tuning thus increases the naturalness reach of the LHC, as our results in section 国 show.

\section{Radiative electroweak symmetry breaking}

Most plots of search reach in the $m_{0}, M_{1 / 2}$ plane found in the literature [3, 12, 17] show a large excluded triangular region, for large $m_{0}$ and small $M_{1 / 2}$. In this region, 
mSUGRA does not have the required properties for radiative electroweak symmetry breaking (REWSB). However, the REWSB constraint, unlike most of the SUSY spectrum, is very sensitive to details of the calculation and input parameters, particularly the value of the running top mass $m_{t}\left(m_{t}\right)$, which must be calculated from the pole top mass in the renormalisation scheme being used. The precise relationship between the running and pole masses depends on the masses of the superparticles [18], and hence on the point in the SUSY parameter space being investigated.

In version 7.14 of ISASUGRA, which was used in [3, 17] to generate SUSY masses and mixings and to obtain the REWSB excluded region, the region above $m_{0} \sim 2 M_{1 / 2}+1 \mathrm{TeV}$ was excluded for an input pole top mass of $174 \mathrm{GeV}$. Here one-loop RGEs were used and minimisation of the scalar potential was performed at scale $M_{Z}$ [21].

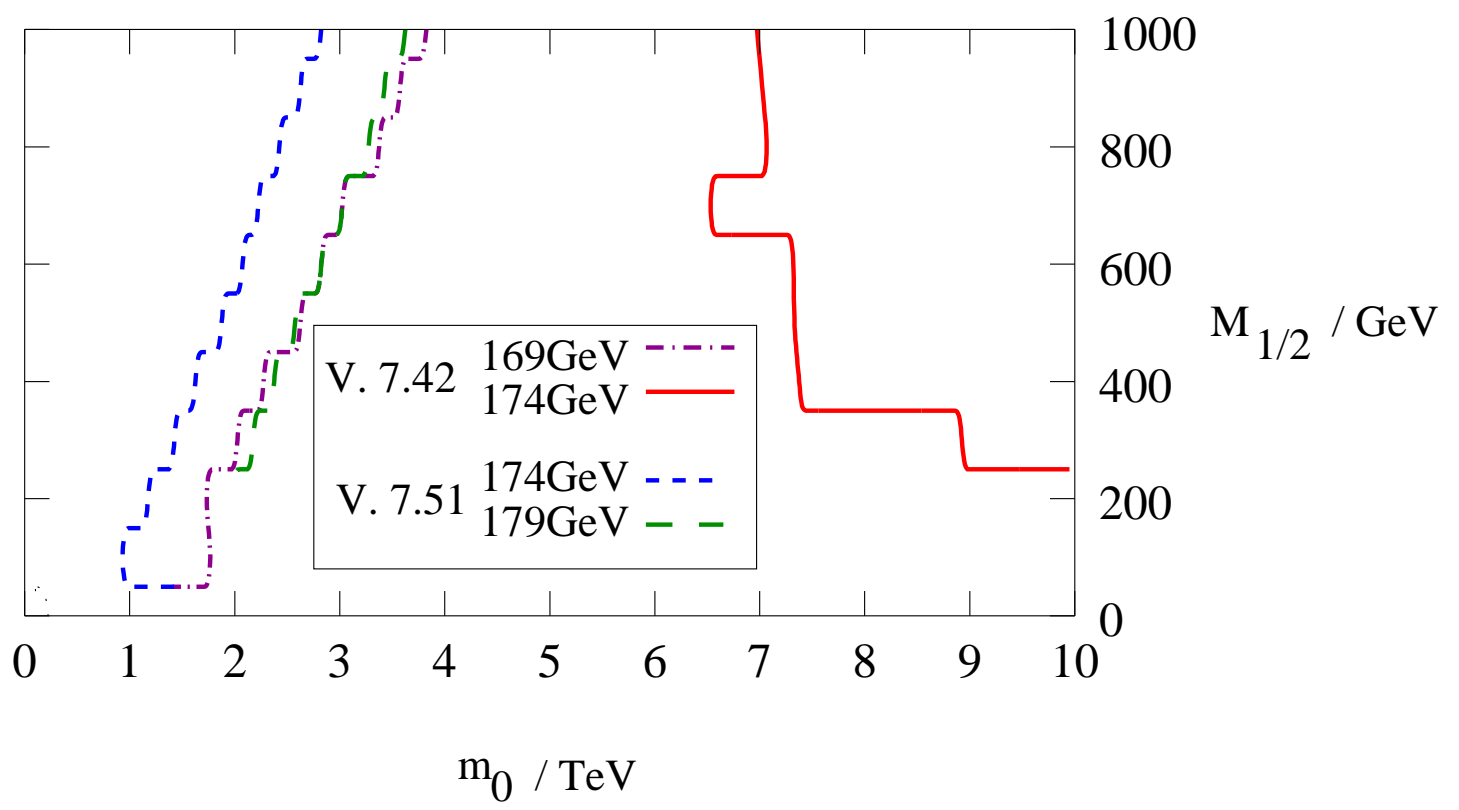

Figure 1: Dependence of the radiative electroweak symmetry breaking excluded region on top mass, according to ISASUGRA versions 7.42 and 7.51 , for $\tan \beta=10, \mu>0$ and $A_{0}=0$.

Between 7.14 and more recent versions [14] such as 7.42, the excluded region shifted to very high $m_{0}$, around $6 \mathrm{TeV}$ for an input pole top mass of $174 \mathrm{GeV}$, as shown in figure 1 . The REWSB constraint therefore no longer provides a useful limit on the values of $m_{0}$ which should be investigated. Here and in later versions, the scalar potential minimisation takes place at $Q=\sqrt{m_{\tilde{t}_{L}} m_{\tilde{t}_{R}}}$ 21]. In the most recent version of ISASUGRA, 7.51, the region has returned once again to low values of $m_{0}$, as shown also in in figure [ [. Here, two-loop corrections to the RGEs and one-loop sparticle mass corrections to the inputs have been added [21].

The REWSB exclusion region was also found using our fine-tuning calculation code, where the physics included was as described in the previous section. The 
dependence of the exclusion region on various aspects of the physics is shown in figure 2. We note again a strong dependence on the top mass, and on the treatment of the running mass and strong coupling.

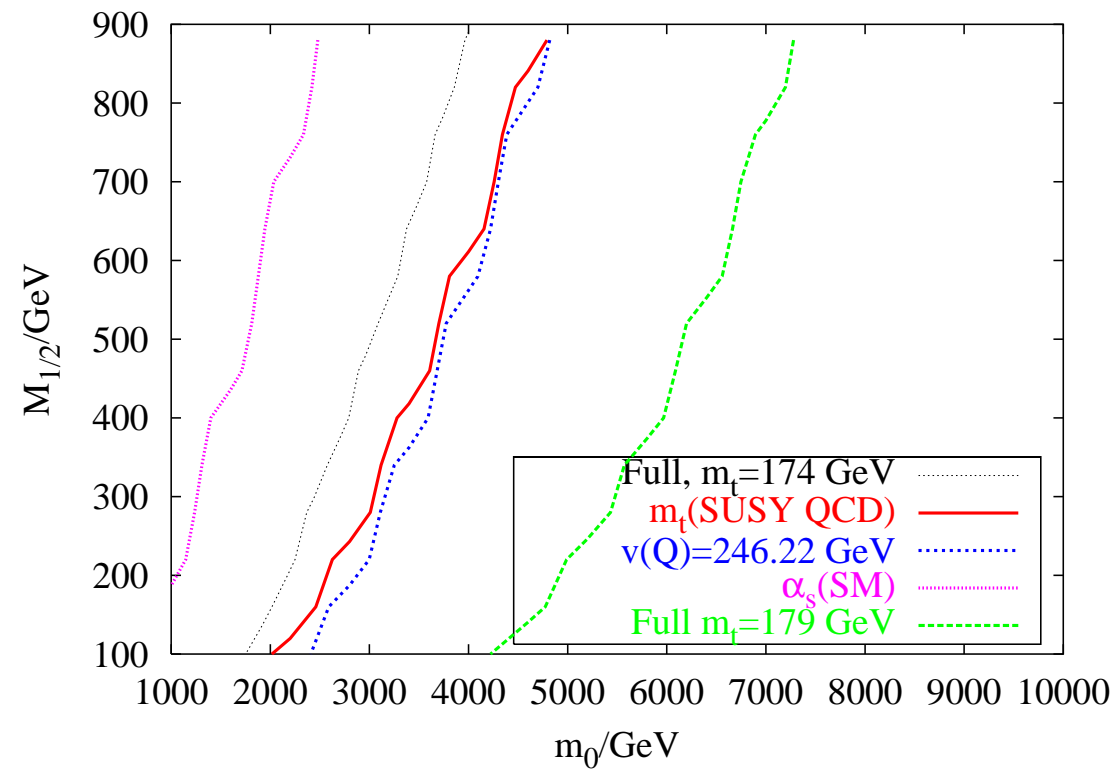

Figure 2: The dependence of the REWSB excluded region in our fine-tuning code on various approximations for $m_{t}=174 \mathrm{GeV}$, except for the curve marked $m_{t}=179 \mathrm{GeV}$. The curves shown are: the full calculations (' $m_{t}=174 \mathrm{GeV}$ ', ' $m_{t}=179 \mathrm{GeV}$ ') and calculations leaving out chargino and neutralino corrections to the running top mass (' $m_{t}$ (SUSY QCD)'), using only a constant value of the Higgs VEV ('v(Q)=246.22 GeV'), and using only standard model corrections to the $\overline{\mathrm{DR}}$ value of $\alpha_{S}\left(M_{Z}\right)$ (' $\alpha_{s}(\mathrm{SM})$ '). Regions to the right of the curves are ruled out by the REWSB constraint.

Since the REWSB constraint is so heavily dependent upon the precise values of input parameters and details of the calculation, there is no reason to suppose that the exclusion region will remain stable under further refinements. Hence we believe it should not be taken as a limit upon the regions of mSUGRA parameter space to be investigated experimentally. Both our own code and ISASUGRA could be subject to large corrections. We therefore use ISASUGRA7.42 to compute the discovery reach, since the allowed region then extends up to values of $m_{0}=10000 \mathrm{GeV}$ (see figure 1). As we shall show, the discovery contour in the allowed region is not so sensitive to the version number or top mass. To produce the REWSB excluded regions displayed in the plots of our results, we used our fine-tuning code with the pole top masses specified in the captions.

\section{Monte-Carlo simulation of LHC discovery reach}

We now turn to the discussion of the LHC mSUGRA search. 
In this study, as in [17], the results of which were used in [3], the SUSY discovery reach in the $m_{0}, M_{1 / 2}$ plane was found through a variety of signals involving hard isolated leptons, jets and missing transverse momentum. These cuts can be applied with a range of cut energies $E_{c u t}$, and are listed below. The cuts represent typical SUSY discovery cuts that might be used at a general-purpose LHC experiment, such as ATLAS [3] or CMS [4].

- Missing transverse momentum $\not{ }_{T}>E_{\text {cut }}$

- Either: At least 2 jets, with pseudo-rapidity $|\eta|<3.2$, and $p_{T}>E_{\text {cut }}$, using a cone algorithm with cone-size 0.7 units of $\eta, \phi$. Or: No jets with $p_{T}>25 \mathrm{GeV}$, using the same jet-finding algorithm.

- Any final state $\left(\mu\right.$ or $e$ ) leptons, with $|\eta|<2.0$ and $p_{T}>20 \mathrm{GeV}$, and lying further in $\eta, \phi$ space than 0.4 units from the centre of any $15 \mathrm{GeV}$ jet with conesize 0.4 , and with less than $5 \mathrm{GeV}$ of energy within 0.3 units of the lepton.

Channels with no leptons, one lepton, two leptons of opposite or the same sign, and three leptons were investigated.

- In the one-lepton channel, an extra cut was imposed to reduce the background due to standard model $W$ decay, involving the transverse mass:

$$
M_{T}=\sqrt{2\left(\left|\mathbf{p}_{\mathbf{l}}\right| \mathbf{p}_{\mathbf{T}} \mid-\mathbf{p}_{\mathbf{l}} \cdot \mathbf{p}_{\mathbf{T}}\right)}>100 \mathrm{GeV}
$$

where $\mathbf{p}_{\mathbf{l}}, \not_{\mathbf{T}}$ are transverse two-component momenta, for the lepton and missing $p_{T}$ respectively.

$$
S_{T}=\frac{2 \lambda_{2}}{\lambda_{1}+\lambda_{2}}>0.2,
$$

where $\lambda_{i}$ are the eigenvalues of the matrix $S_{i j}=\Sigma_{i j} p_{i} p_{j}$, the sum being taken over all detectable final state particles, and $p_{i}$ being the two-component transverse momentum of the particle. $S_{T}$ is often called the transverse circularity or transverse sphericity.

The search channels used are shown in table 11.

The mSUGRA events were simulated by employing the ISASUSY part of the ISAJET7.42 package [14] to calculate sparticle masses and branching ratios, and HERWIG6.1 [15, 16] to simulate the events themselves. The expected SUSY signal was generated, together with the backgrounds due to standard model top anti-top and $W$ plus jet events. The $W$ background was generated using events with one of the jets produced in the hard subprocess, and the rest in QCD parton cascades. This produces an underestimate of the $W$ background, as discussed in [22], and the results were rescaled accordingly. 
The discovery limit is set at values of $m_{0}$ and $M_{1 / 2}$ where $S / \sqrt{B}>5$ and $S>10$ where $\mathrm{S}$ and $B$ are the expected number of events in the the SUSY signal and total background. The former constraint is an approximation to the requirement that the total number of observed events in some experiment will be significantly above the background at the $5 \sigma$ level.

For each value of $E_{c u t}$, the minimum final state transverse momentum in the hard subprocess was selected to obtain some events passing the cuts, within realistic computer time con-

\begin{tabular}{|c|c|c|}
\hline Jets & Leptons & Label \\
\hline$>1$ & 0 & $\mathrm{j} 0$ \\
& 1 & $\mathrm{j} 1$ \\
& 2 (Opposite sign) & $\mathrm{jos}$ \\
& 2 (Same sign) & $\mathrm{jss}$ \\
& 3 & $\mathrm{j} 3$ \\
\hline 0 & 2 & $\mathrm{v} 2$ \\
& 3 & $\mathrm{v} 3$ \\
\hline
\end{tabular}

Table 1: Channels in which the SUSY discovery reach has been investigated. straints. Where the minimum transverse momentum had to be increased above $E_{c u t}$, results from the j0 channel were used to obtain linear correction factors to compensate for the resulting underestimate of the background.

In order to find the hard leptons, and to determine $\mathbf{p}_{\mathbf{T}}$ and $S_{T}$, the Monte Carlo output was examined directly, particles with $|\eta|>5.0$ being ignored. For the jet count and lepton isolation check, a calorimeter simulation was used, with hadronic resolution of $70 \% / \sqrt{E}$, electromagnetic resolution of $10 \% / \sqrt{E}$ and extending to pseudo-rapidities up to 5.0. These parameters have been selected to simulate a general-purpose experiment at the LHC.

Calculations were made for mSUGRA with $A_{0}=0, \tan \beta=10$, pole top mass $174 \mathrm{GeV}, \mu>0$ and $\mathcal{L}=10 \mathrm{fb}^{-1}$ of integrated luminosity. The integrated luminosity chosen is equivalent to one year of running in the low luminosity mode, $10^{33} \mathrm{~cm}^{-2}$ $\mathrm{s}^{-1}$. We note that the LHC experiments expect to collect around $300 \mathrm{fb}^{-1}$ of data.

A low-statistics calculation of the discovery reach with a top mass of $179 \mathrm{GeV}$ was also made, to determine whether the reach is independent of the top mass. There is a small decrease in the production cross section for $t \bar{t}$ background with increasing top mass, and a compensating increase in the energy of the resulting leptons and jets, which causes a greater proportion to pass the selection cuts. Thus for this study the background could safely be assumed to be independent of the top mass.

\section{Results}

Preliminary results on the naturalness reach of the LHC for the j1 channel, with $E_{\text {cut }}=400 \mathrm{GeV}$, were presented in [23]. The top Yukawa coupling was not included in the definition of fine-tuning. Here we obtain the discovery limit in all the channels in table 1, for several values of $E_{c u t}$, and with both definitions of fine-tuning (with/without the top Yukawa coupling). 
Table 2 shows the background for each generated channel, the total background, and the resulting expected number of supersymmetry events required to fulfill the discovery criteria detailed above. No discernible background in the jet veto channels $(\mathrm{v} 2, \mathrm{v} 3)$ was obtained.

\begin{tabular}{|c|c|c|c|}
\hline Channel & $E_{\text {cut }} / \mathrm{GeV}$ & $\begin{array}{c}\text { Total no of } \\
\text { background events }\end{array}$ & $\begin{array}{c}\text { Required no of } \\
\text { SUSY Signal events }\end{array}$ \\
\hline j0 & 100 & $5.3 \times 10^{5}$ & $3.6 \times 10^{3}$ \\
\hline j0 & 200 & $5.2 \times 10^{4}$ & $1.4 \times 10^{3}$ \\
\hline j0 & 300 & $7.4 \times 10^{3}$ & 431 \\
\hline j0 & 400 & $1.6 \times 10^{3}$ & 201 \\
\hline j0 & 500 & 451 & 107 \\
\hline $\mathrm{j} 1$ & 100 & $1.3 \times 10^{4}$ & 579 \\
\hline $\mathrm{j} 1$ & 200 & 394 & 102 \\
\hline $\mathrm{j} 1$ & 300 & $35 *$ & 29 \\
\hline $\mathrm{j} 1$ & 400 & 4 & 10 \\
\hline j1 & 500 & 1 & 10 \\
\hline j3 & 100 & $36^{*}$ & 30 \\
\hline j3 & 200 & $20 *$ & 22 \\
\hline j3 & 300 & $<1^{*}$ & 10 \\
\hline $\mathrm{j} 3$ & 400 & $<1$ & 10 \\
\hline j3 & 500 & $<1$ & 10 \\
\hline$\overline{\text { jss }}$ & 100 & $115^{*}$ & 54 \\
\hline jss & 200 & $7^{*}$ & 13 \\
\hline jss & 300 & $4^{*}$ & 10 \\
\hline jiss & 400 & 4 & 10 \\
\hline jss & 500 & 2 & 10 \\
\hline jos & 100 & $7.4 \times 10^{3} *$ & 431 \\
\hline jos & 200 & $173 *$ & 65 \\
\hline jos & 300 & $12 *$ & 17 \\
\hline jos & 400 & 5 & 11 \\
\hline jos & 500 & 2 & 10 \\
\hline
\end{tabular}

Table 2: Total background for SUSY discovery channels. Also shown is the expected number of SUSY events required for discovery. Backgrounds marked with an asterisk (*) have been corrected for the use of a minimum subprocess $p_{T}$ greater than $E_{c u t}$, as described in section 4 .

For each of the channels, and each value of $E_{\text {cut }}$, a plot has been used to determine the fine-tuning reach with both definitions of fine-tuning. The fine-tuning reach is the largest fine tuning where the fine-tuning contour is completely within the discovery limit, for $m_{0}<4000 \mathrm{GeV}$. The values of $E_{\text {cut }}$ giving the best fine-tuning reach, and 


\begin{tabular}{|c||c|c||c|c||c|c||c|c||c|c|}
\hline Cut Type & $E_{\text {cut }}$ & $c$ & $E_{\text {cut }}$ & $c^{\prime}$ & $E_{\text {cut }}$ & $c_{h_{t}}$ & $E_{\text {cut }}$ & $c_{h_{t}}^{\prime}$ & $E_{\text {cut }}$ & $M_{1 / 2}$ limit \\
\hline j0 & 300 & 120 & 300 & 230 & 400 & 375 & 400 & 375 & 200 & 350 \\
j1 & 400 & 210 & 400 & 260 & 400 & 500 & 400 & 550 & 300 & 460 \\
jos & 300 & 85 & 300 & 190 & 300 & 240 & 300 & 270 & 200 & 300 \\
jss & 200 & 110 & 200 & 215 & 200 & 240 & 200 & 270 & 200 & 350 \\
j3 & 100 & 70 & 100 & 130 & 100 & 150 & 100 & 155 & 100 & 280 \\
\hline
\end{tabular}

Table 3: Values of the cut on missing momentum and jet energy which give the best fine-tuning limits $c$ and $c_{h t}$, and the $M_{1 / 2}$ reach for large $m_{0}>4000 \mathrm{GeV}$, for each of the supersymmetry discovery channels. $c$ is the fine-tuning limit as defined in the text, and $c_{h_{t}}$ is the same with the fine-tuning with respect to the top Yukawa coupling included, using a top mass of $174 \mathrm{GeV}$ to calculate the REWSB excluded region and fine-tuning. $c^{\prime}$ refers to the same quantities for a top mass of $179 \mathrm{GeV} . E_{c u t}$ and $M_{1 / 2}$ are given in $\mathrm{GeV}$.

the corresponding reach, are shown in table 3. The results using $m_{t}=174 \mathrm{GeV}$ and $m_{t}=179 \mathrm{GeV}$ to calculate the fine-tuning and REWSB exclusion are given. $E_{c u t}=100,200,300,400$ and $500 \mathrm{GeV}$ were investigated. No results can be presented for the jet veto channels, as the number of events in these channels is too small. The reach in these channels could be obtained by considering specific SUSY processes, but is expected to be very limited.

Figures 3 and 4 show the best obtainable discovery limit using the channel providing the best reach, the $E_{c u t}=400 \mathrm{GeV}$ j1 channel, as a dashed line in the $\left(m_{0}, M_{1 / 2}\right)$ plane, and the naturalness density without and with the top Yukawa coupling included, respectively. The resulting fine-tuning limits, as defined above, are 210 and 500 units. Excluded regions due to experimental limits on the chargino mass, and due to the cosmological requirement that the LSP be neutral, are included in the figures. The current limit on the light Higgs mass does not exclude any of the region of mSUGRA parameter space illustrated, due to the large value of $\tan \beta$ used here.

Figure 5 shows consistency of our results for $m_{0}<2 \mathrm{TeV}$ in the $\mathrm{j} 1$ channel with those obtained using ISAJET 7.14 in [17]. The same figure shows the discovery reach calculated with ISAJET7.51 (and lower statistics). We see that that the differences in approximation between the various ISAJET versions which shifted the REWSB exclusion, as discussed in section 3, do not appreciably alter the discovery reach.

Also shown in figure 5 is the discovery reach for a $1 \sigma$ increase in the top mass, $m_{t}=179 \mathrm{GeV}$. Unlike the REWSB exclusion region, the discovery contours are almost identical. We may therefore use our high-statistics $m_{t}=174 \mathrm{GeV}$ ISAJET 7.42 discovery contour to calculate the fine-tuning reach for $m_{t}=179$. As shown in table 3 and figure 6, the reach extends to a fine-tuning value of 260 .

For $m_{0}>4000 \mathrm{GeV}$ the discovery reach in $M_{1 / 2}$ becomes roughly independent of $m_{0}$. Here, the SUSY processes involved are dominated by the gauginos, the squark masses being very high (larger than $3 \mathrm{TeV}$ ), as illustrated in figure 7. This figures 


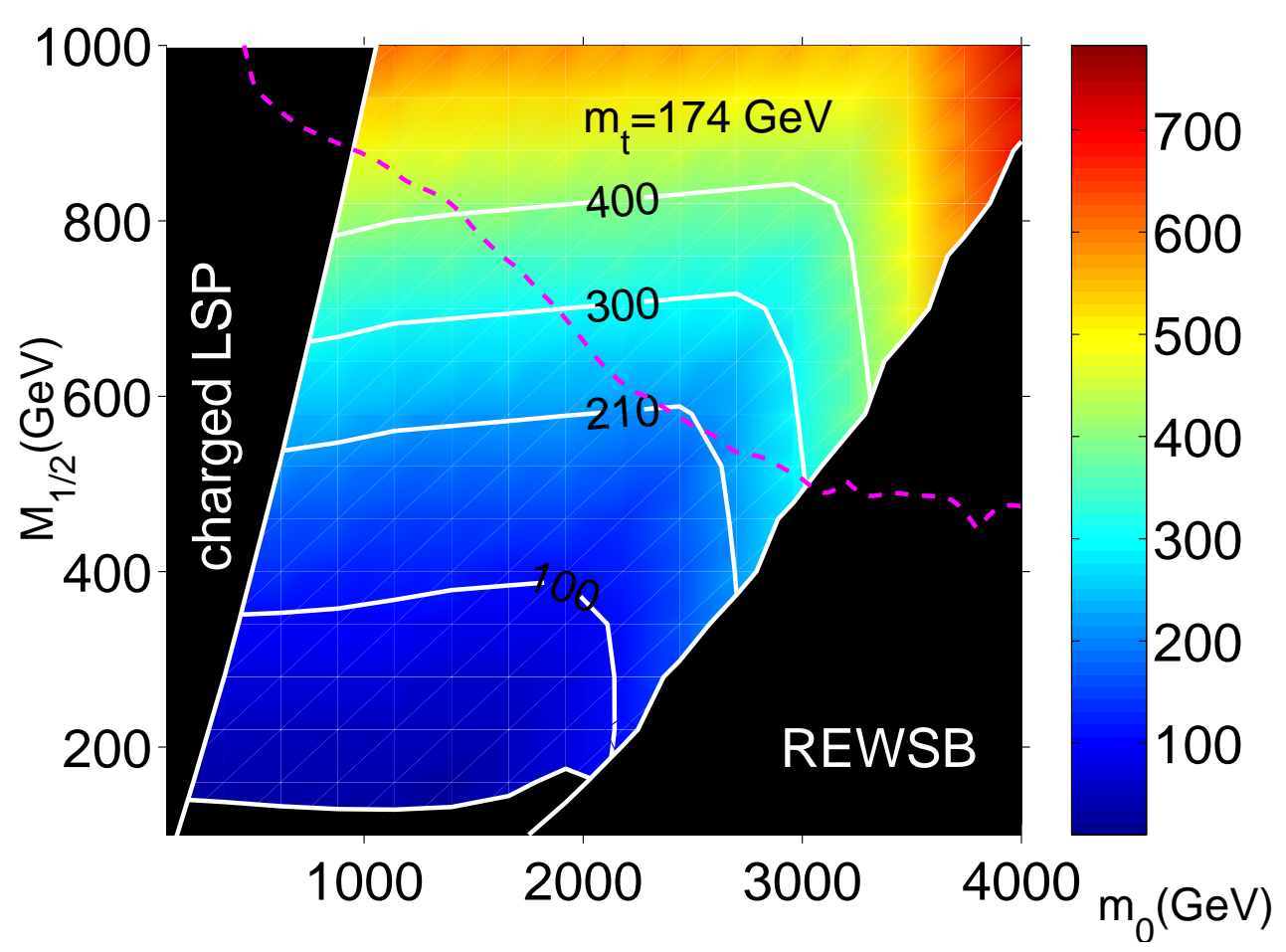

Figure 3: Naturalness reach at the LHC for $A_{0}=0, \tan \beta=10, \mu>0, m_{t}=174 \mathrm{GeV}$ in minimal SUGRA. The fine-tuning is represented by the background density, as measured by the bar on the right. White contours of fine-tuning are also presented. Fine tuning with respect to the top Yukawa coupling is neglected. The dashed line is the LHC expectation SUSY discovery contour in the $\mathrm{j} 1$ channel described in the text for a luminosity of $\mathcal{L}=$ $10 \mathrm{fb}^{-1}$, and with $E_{c u t}=400 \mathrm{GeV}$. The excluded region, filled black, along the left hand side of the plot is due to the cosmological requirement that the lightest supersymmetric particle be neutral, while that along the bottom of the plot is due to the experimental lower bound on the chargino mass. The excluded region on the right hand side of the plot is due to lack of radiative electroweak symmetry breaking.

also shows that scalar production, as a fraction of total SUSY processes, decreases at high $m_{0}$, being typically a percent around $m_{0} \sim 3 \mathrm{TeV}$. However, it is clear that at values of $M_{1 / 2}$ lower than the SUSY discovery contour but at $m_{0}>2 \mathrm{TeV}$, it is possible to produce scalar SUSY particles at the LHC. A discussion of how to attempt to obtain a limit on the region of mSUGRA parameter space where the squarks in particular (rather than SUSY in general) can be discovered is beyond the scope of this paper.

We terminate the calculation of the fine-tuning and search-reach at $m_{0}=4000 \mathrm{GeV}$, since for higher values of $m_{0}$ the $M_{1 / 2}$ discovery reach gives an adequate representation of the overall SUSY discovery power, through the gauginos, in each channel. This expression of the reach is also shown in table 3 . 


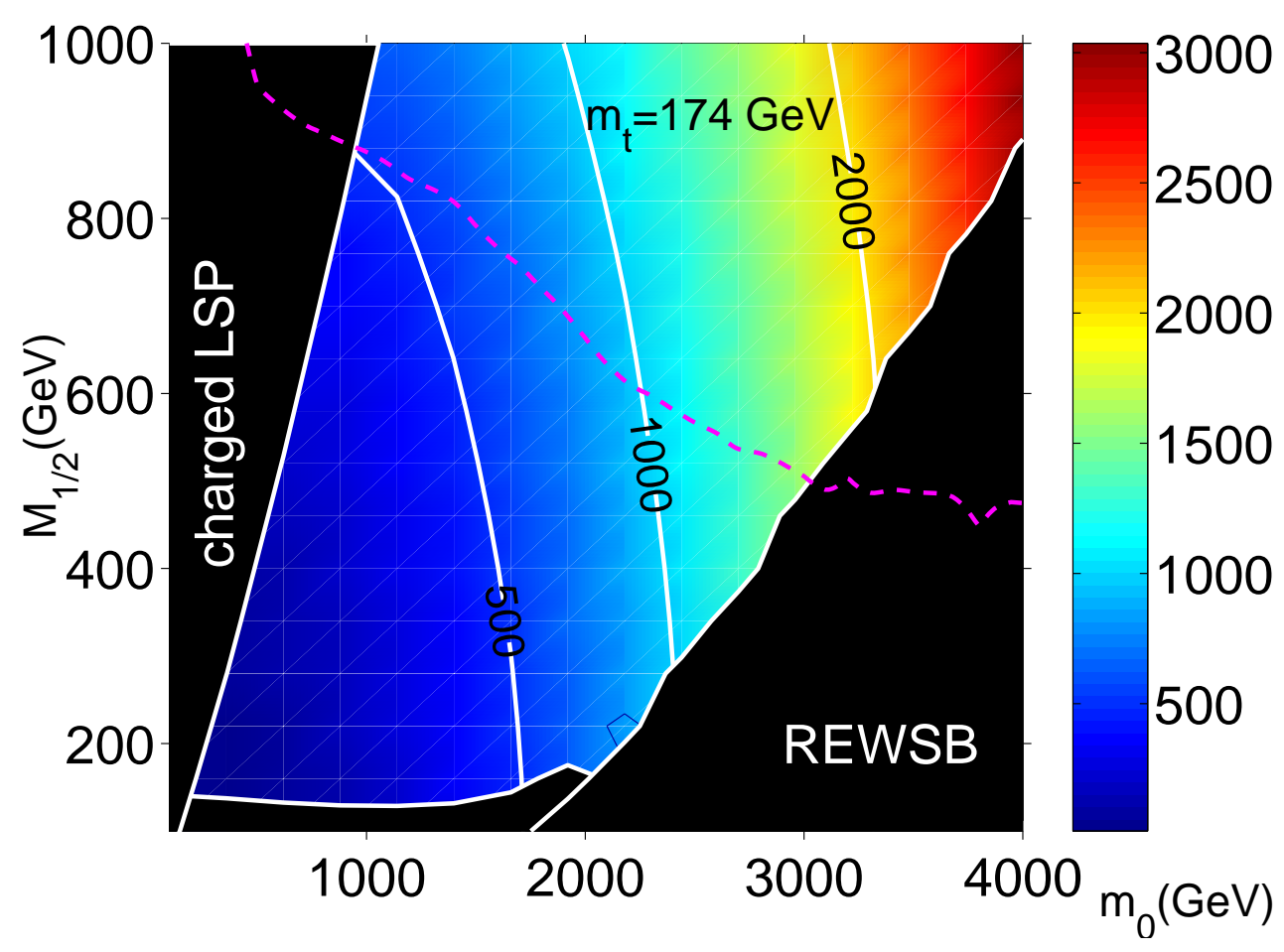

Figure 4: As in figure 3, but with fine tuning with respect to the top Yukawa coupling included.

\section{Summary}

In this paper, we have obtained the discovery reach of the LHC into mSUGRA parameter space, using the new supersymmetry routines in HERWIG. Where our investigation repeats the calculation of [17], this provides a useful check on the consistency of the two Monte-Carlos. In addition, our use of the latest software for calculating the mSUGRA spectrum updates the old results, and allows us to move into the region of high scalar masses $m_{0}$. It has been suggested that a focus point gives this region increased naturalness, and the extent to which radiative electroweak symmetry breaking excludes high $m_{0}$ remains uncertain, so this region should be explored thoroughly. We demonstrate that even for arbitrarily high $m_{0}$, the standard SUSY searches at the LHC can discover supersymmetry, through events involving gauginos, provided they are not too heavy $\left(M_{1 / 2}<460\right)$.

We have introduced the possibility of using fine-tuning as a quantitative way to compare the discovery reach of various channels. Fine-tuning can provide physicists with a quantitative measure of discomfort with a theory, which increases as the experimental bounds are improved. Since it is this disquiet which leads, in the end, to the abandonment of a theory such as supersymmetry, the fine-tuning reach represents the potential of these discovery channels for removing mSUGRA from 


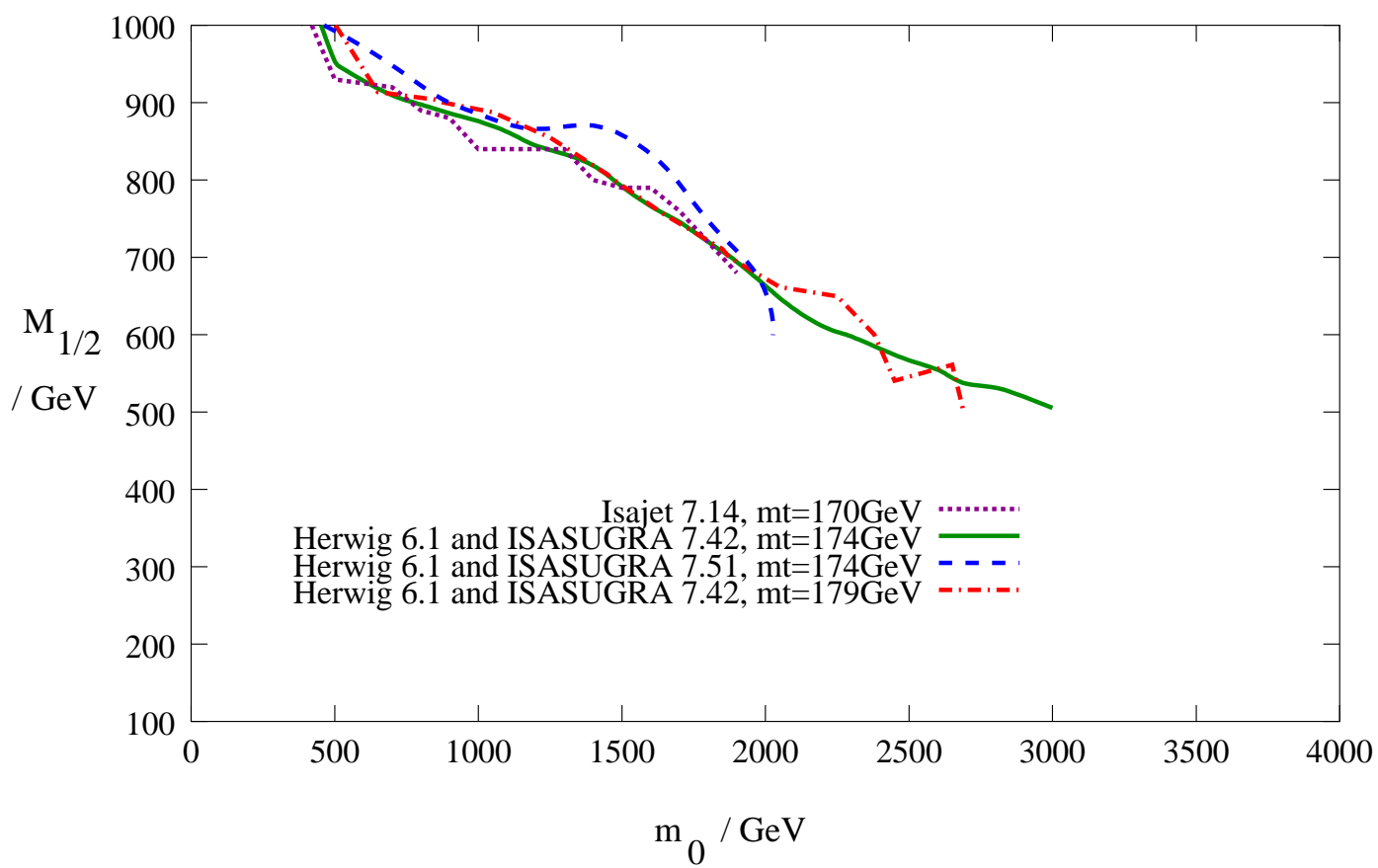

Figure 5: Reach at the LHC for $A_{0}=0, \tan \beta=10, \mu>0$, in minimal supergravity. Solid: HERWIG results with $m_{t}=174 \mathrm{GeV}$, with ISASUGRA version 7.42 to generate the SUSY spectrum and decays. Dot-dashed: The same with $m_{t}=179 \mathrm{GeV}$. Short-dashed: ISAJET results of [14] (with $m_{t}=170 \mathrm{GeV}$ ). Long-dashed: HERWIG results with $m_{t}=174 \mathrm{GeV}$, using ISASUGRA version 7.51 to generate the SUSY spectrum and decays.

the list of candidate theories for physics beyond the standard model. The work could be repeated using some different high-energy unification assumptions, instead of mSUGRA. The mSUGRA reach into $A_{0}$ and $\tan \beta$ could also be investigated.

The best fine tuning reach is found in a mono-leptonic channel, where for $\mu>$ $0, A_{0}=0, \tan \beta=10$ (within the focus point region), and $m_{t}=174 \mathrm{GeV}$, all points in the $m_{0}, M_{1 / 2}$ plane with $m_{0}<4000 \mathrm{GeV}$ and a fine tuning measure up to 210 (500) are covered by the search, where the definition of fine-tuning excludes (includes) the contribution from the top Yukawa coupling. For $m_{0}>4000$, all values of $M_{1 / 2}<$ $460 \mathrm{GeV}$ are covered by the search.

\section{Acknowledgments}

Part of this work was produced using the Cambridge University High Performance Computing Facility. BCA would like to thank K. Matchev for valuable discussions on checks of the numerical results. JPJH would like to thank C.G. Lester for useful discussions and CERN Theory Division for hospitality. The authors also thank G.G. Ross for helping to motivate this study.

This work was funded by the U.K. Particle Physics and Astronomy Research Council. 


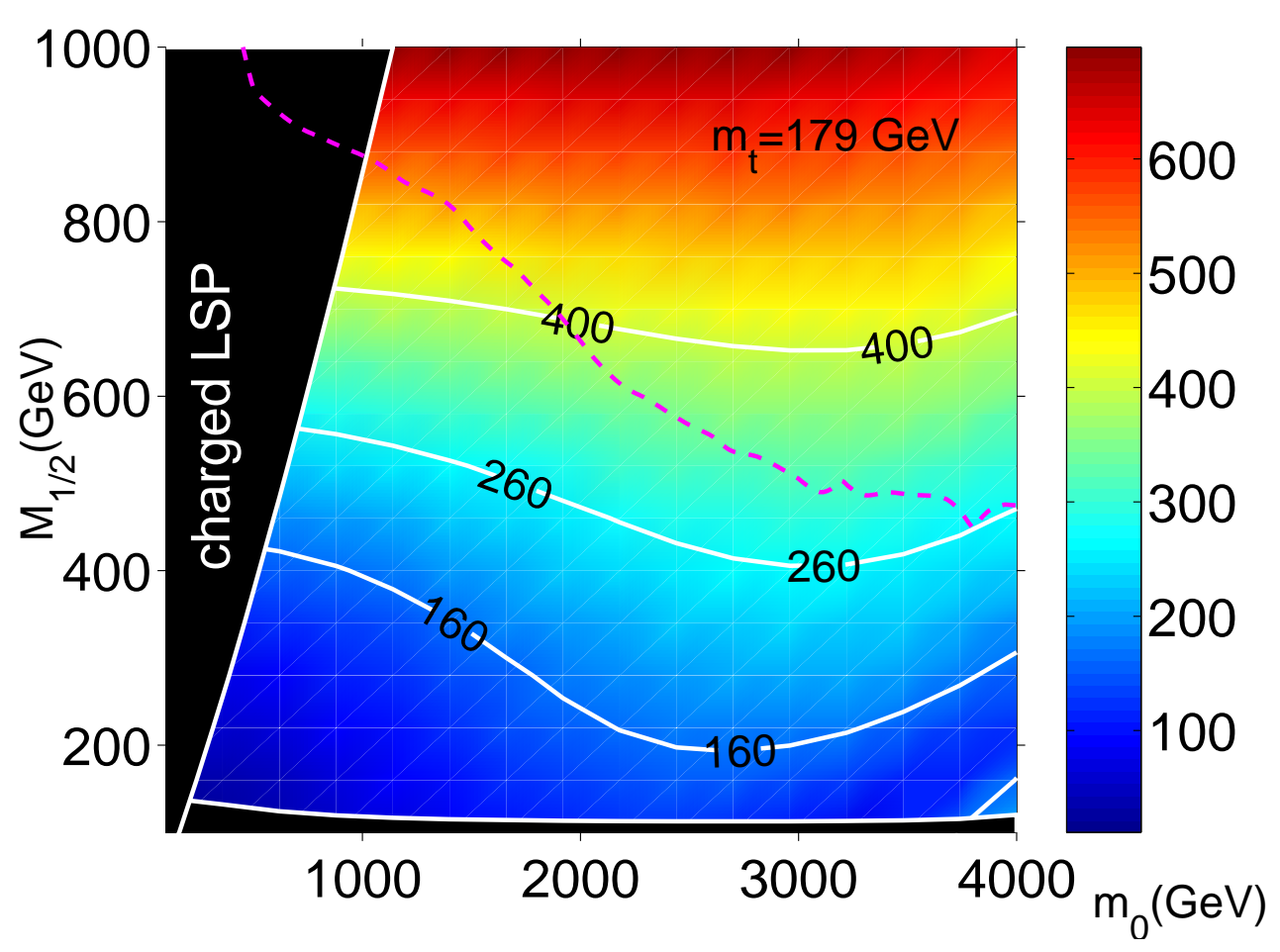

Figure 6: As in figure 3, but with a top mass of $179 \mathrm{GeV}$ used to calculate the fine-tuning and REWSB excluded region.

\section{References}

[1] C. Kolda and H. Murayama, "The Higgs mass and new physics scales in the minimal standard model", hep-ph/0003170.

[2] H. E. Haber, "Introductory low-energy supersymmetry", hep-ph/9306207.

[3] ATLAS Collaboration, Detector and Physics Performance TDR, Volume II, Technical Report CERN/LHCC 99-15, (1999) CERN.

[4] CMS Collaboration, Technical Proposal, http://cmsinfo.cern.ch/TP/TP.html.

[5] H. Baer, C. Chen, M. Drees, F. Paige and X. Tata, "Probing minimal supergravity at the CERN LHC for large tan $\beta "$, Phys. Rev. D 59 (1999) 055014 hep-ph/9809223

[6] S. Dimopoulos and G. F. Giudice, "Naturalness constraints in supersymmetric theories with nonuniversal soft terms", Phys. Lett. B 357 (1995) 573 hep-ph/9507282.

[7] See for example R. Barbieri and A. Strumia, Phys. Lett. B 433 (1998) 63; B. de Carlos and J.A. Casas, Phys. Lett. B 320 (1993) 320.

[8] G. W. Anderson and D. J. Castano, "Naturalness and superpartner masses or when to give up on weak scale supersymmetry", Phys. Rev. D 52 (1995) 1693 hep-ph/9412322. 


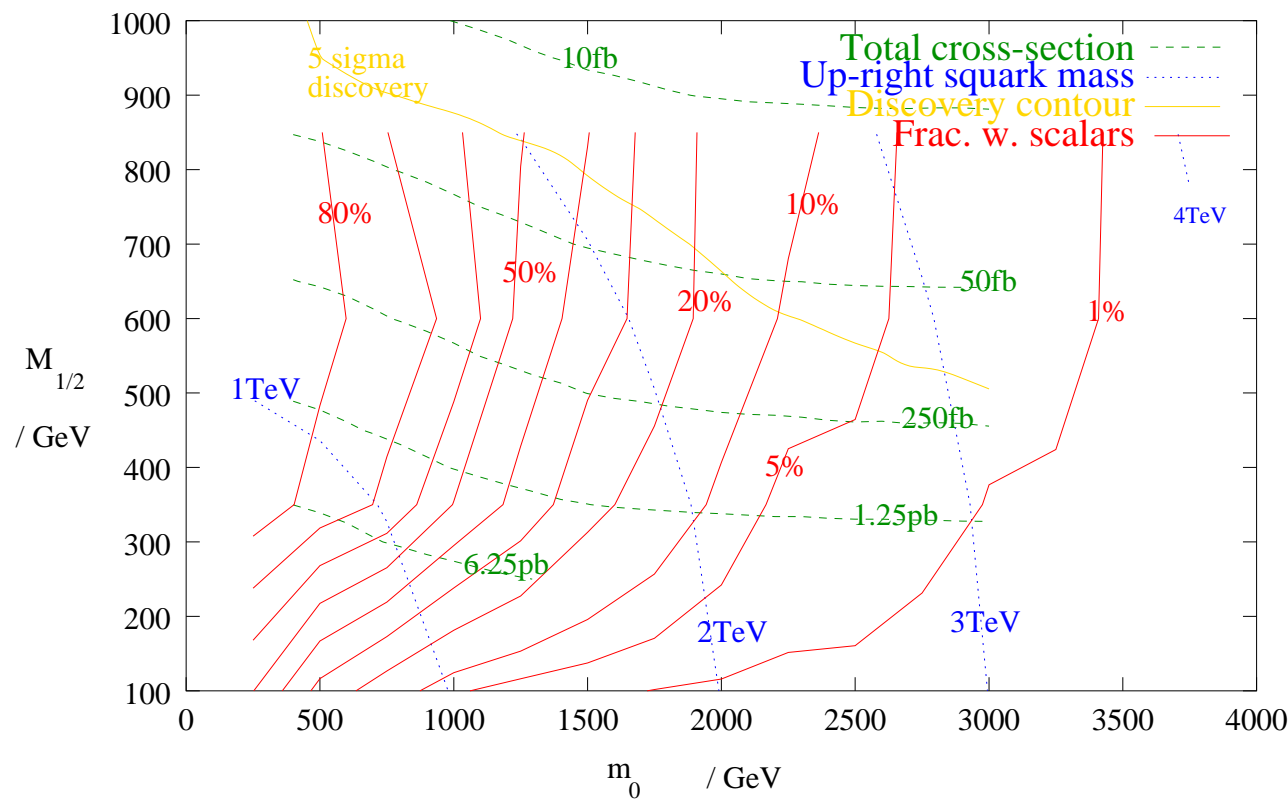

Figure 7: Scalar production in mSUGRA. The contours represent the total SUSY crosssection, the mass of a typical squark, (the up-right), the overall SUSY discovery contour, and the fraction of SUSY processes involving a squark or slepton.

[9] B. de Carlos and J. A. Casas, "One loop analysis of the electroweak breaking in supersymmetric models and the fine tuning problem", Phys. Lett. B 309 (1193) 320 hep-ph/9303291.

[10] M. Bastero-Gil, G. L. Kane and S. F. King, "Fine-tuning constraints on supergravity models", Phys. Lett. B 474 (2000) 103 hep-ph/9910506.

[11] D. Wright, "Naturally nonminimal supersymmetry", hep-ph/9801449.

[12] J. L. Feng, K. T. Matchev and T. Moroi, "Focus points and naturalness in supersymmetry", Phys. Rev. D 61 (2000) 075005 hep-ph/9909334.

[13] J. L. Feng, K. T. Matchev and T. Moroi, "Multi-TeV scalars are natural in minimal supergravity", Phys. Rev. Lett. 84 (2000) 2322 hep-ph/9908309.

[14] F. E. Paige, S. D. Protopescu, H. Baer and X. Tata, "ISAJET 7.40: A Monte Carlo event generator for $\mathrm{p} \mathrm{p}$, anti-p p, and $e^{+} e^{-}$reactions", hep-ph/9810440.

[15] G. Corcella et al., "HERWIG 6.1 release note", hep-ph/9912396.

[16] G. Marchesini, B. R. Webber, G. Abbiendi, I. G. Knowles, M. H. Seymour and L. Stanco, "HERWIG: A Monte Carlo event generator for simulating hadron emission reactions with interfering gluons. Version 5.1 - April 1991", Comput. Phys. Commun. 67 (1992) 465. 
[17] H. Baer, C. Chen, F. Paige and X. Tata, "Signals for minimal supergravity at the CERN Large Hadron Collider. 2: Multi-lepton channels", Phys. Rev. D 53 (1996) 6241 .

[18] J. A. Casas, J. R. Espinosa and H. E. Haber, "The Higgs mass in the MSSM infrared fixed point scenario", Nucl. Phys. B 526 (1998) 3 hep-ph/9801365.

[19] D.M. Pierce, J.A. Bagger, K.T. Matchev and R.J. Zhang, "Precision corrections in the minimal supersymmetric standard model", Nucl. Phys. B 491 (1997) 3 hep-ph/9606211.

[20] A. Romanino and A. Strumia, "Are heavy scalars natural in minimal supergravity?", hep-ph/9912301.

[21] H. Baer, private communication.

[22] W.T. Giele el al., "W Boson plus Multijets at Hadron Colliders: HERWIG Parton Showers vs Exact Matrix Elements", Proceedings of SNOWMASS 1990.

[23] B.C. Allanach et al., "Report of the beyond the standard model working group of the 1999 UK Phenomenology Workshop on Collider Physics (Durham)", J. Phys. G: Nucl. Part. Phys. 26 (2000) 1 hep-ph/9912302. 